\title{
Application of Predictive Dietary Guidance in Radiotherapy Nursing of Esophageal Cancer
}

\author{
Qiuping Zhang, Guihong Xing, Dejing $\mathrm{Xu}^{*}$ \\ Department of Radiotherapy, Jiangsu Cancer Hospital (Jiangsu Institute of Cancer Research, the Affiliated Cancer Hospital \\ of Nanjing Medical University), Nanjing 210009, Jiangsu Province, China
}

*Corresponding author: Dejing Xu, 94584891@qq.com

Copyright: (c) 2022 Author(s). This is an open-access article distributed under the terms of the Creative Commons Attribution License (CC BY 4.0), permitting distribution and reproduction in any medium, provided the original work is cited.

\begin{abstract}
Objective: To explore the effect of predictive diet guidance in radiotherapy nursing of esophageal cancer. Methods: 96 patients with esophageal cancer in our hospital (from January 2019 to January 2021) were randomly divided into group A and group B. Group A (48 cases) received routine nursing, and group B (48 cases) received predictive dietary guidance on the basis of group A. The incidence of adverse reactions, clinical indicators and quality of life were compared between the two groups. Results: After nursing, the incidence of adverse reactions of radiotherapy in group B was lower than that in group A, the clinical indexes in group B were higher than those in group A, and the quality of life in group B was higher than that in group A $(\mathrm{P}<0.05)$. Conclusion: For esophageal cancer patients undergoing radiotherapy, the implementation of predictive diet guidance can effectively reduce complications, improve the quality of life of patients, and improve nursing satisfaction. The effect is ideal, which is worthy of widespread application.
\end{abstract}

Keywords: Predictive dietary guidance; Esophageal cancer; Radiotherapy; Application effect

Online publication: January 19, 2022

\section{Introduction}

Esophageal cancer is a term that refers to malignant tumors that arise from the esophageal epithelium and are characterized by the following symptoms: Choking, foreign body sensations, and other symptoms may occur when swallowing food, and may be accompanied by retrosternal discomfort or evident swallowing problems, which can lead to eating difficulties and diminished appetite ${ }^{[1]}$. Furthermore, malignant tumors can cause a fast metabolism, increased protein and fat intake, and an increase in the amount of energy required by the body. If this continues for a long time, patients may develop malnutrition, body tolerance, and other disorders that make clinical therapy difficult. According to the findings, predictive dietary counselling can successfully enhance nutritional indicators and improve quality of life for patients with esophageal cancer. The specific implementation methods and results are summarized as follows.

\section{Materials and methods}

\subsection{General information}

From January 2019 to January 2021, 96 patients with esophageal cancer were randomly selected for study. According to the random number table, 96 patients were divided into group A and group B. There were 48 patients in group A, including 25 males and 23 females, aged from 38 to 76 years old, with an average age of $(56.47 \pm 7.47)$ years old. There were 48 cases in group B, including 26 males and 22 females, aged from 39 to 77 years old, with an average age of $(57.51 \pm 7.48)$ years old. There was no difference between groups 
$(\mathrm{P}>0.05)$.

\subsubsection{Inclusion criteria}

(1) The patient was diagnosed as esophageal cancer by medical examination and met the radiotherapy standard.

(2) Patients without communication disorder and mental history.

(3) The situation was explained to patients and their families and they signed informed consent forms.

2.1.2. Exclusion criteria

(1) Patients with visceral diseases, such as: heart, liver, kidney, etc.

(2) Patients with infectious diseases.

(3) Patients with extensive metastasis of cancer cells.

(4) Patients with survival less than 6 months after clinical evaluation.

(5) Patients who do not cooperate with this study and quit halfway.

\subsection{Methods}

\subsubsection{Group A received routine nursing, and the specific operation methods were as follows}

After the patient is admitted to the hospital, we should first assist the patient to complete the relevant examination, and explain the disease-related knowledge, the purpose and precautions of radiotherapy to the patient. For patients with negative emotions such as fear and anxiety, they should be given psychological counseling in time to improve the patient's adverse psychology. During radiotherapy, we should pay attention to the patient's vital signs and observe whether the patient has adverse reactions. And after radiotherapy, we should give patients routine medication guidance, inform them of the correct use of drugs and precautions, give patients routine dietary guidance, inform patients of some dietary taboos, etc.

\subsubsection{Group B was given predictive dietary guidance on the basis of routine nursing, and specific operation methods were as follows}

\subsubsection{Proactive inquiry and nutritional assessment}

First, the nursing staff should actively communicate with the patient, ask the patient whether the diet has been changed before and after radiotherapy and chemotherapy, whether there is discomfort during eating, and how the patient's diet is after illness, and preliminarily understand the patient's eating habits and diet. And the patient's height, weight, body mass index and nutritional indicators should be screened within 24 hours of admission, and the patient's nutritional risk should be assessed. Then, according to the patient's dietary preferences and nutritional status, a diet plan can be made for the patient, and feeding cups can be used, and the patient's food intake and body mass index can be observed and recorded every day, and then constantly adjust and improve the diet plan.

\subsubsection{Dietary guidelines}

(1) Before radiotherapy: First of all, it should be given priority to with easily digestible and soft food, which should be a light diet, and should be rich in vitamins, protein, high calorie and other food, and then maintain the normal body needs of patients. Due to patients may have difficulties in eating, pain and other situations, the nursing staff should comfort the patients at this time, and can give $2 \%$ lidocaine spray before the patients eat according to the doctor's advice, which is conducive to the patients' eating. The nursing staff can recommend that the family members of the patients carry out with the patients, so as to create a good diet atmosphere for the patients.

(2) During radiotherapy: Firstly, oral cleaning should be carried out before and after eating, and the 
esophagus should be kept clean. After eating, it is necessary to drink a moderate amount of warm boiled water to achieve the effect of flushing esophagus, which can effectively reduce the discomfort of patients with radiotherapy. And for patients with radioactive esophagitis, the diet should be based on semi-liquid food. Food should not be overheated, should be warm and cool appropriate, and should be given at the same time with gargle physiological saline and vitamin C. Nasal feeding can be given to patients who cannot take food through the mouth or who have hyperemia and edema of the esophageal mucosa, and nutritional support can be given to patients when necessary. On the first day of nutritional support, $250 \mathrm{ml}$ normal saline should be pumped, and $500 \mathrm{ml}$ nutrient solution should be given to the patient on the second day. On the third and fourth days, $1000 \mathrm{ml}$ nutrient solution should be given to the patient, and on the fifth and eighth days, $1500 \mathrm{ml}$ nutrient solution can be gradually increased. When giving nutritional support to patients, the infusion speed of nutrient solution should be adjusted according to the patient's tolerance, and the patient's head should be raised to avoid reflux.

(3) After radiotherapy: Firstly, the nursing staff should reasonably plan the patient's diet plan according to the patency of the patient's esophagus, and should tell the patient and his family that the patient should not eat hard and slag food after radiotherapy. They also should tell the patient to chew carefully and not swallow, which can effectively avoid damage to the patient's esophagus, and after eating, the patient should maintain standing or sitting position to avoid food reflux.

\subsection{Observation indexes}

(1) The incidence of adverse reactions of radiotherapy in the two groups was observed

(2) It mainly includes: pulmonary infection, electrolyte imbalance, weight loss, etc. Total incidence rate = (total number of cases occurred / total cases number) $\times 100 \%$.

(3) Observe the clinical indexes of the two groups

(4) It mainly includes: Serum albumin, hemoglobin, lymphocyte coefficient, etc.

(5) Observe the quality of life of the two groups

(6) SF-36 health measurement table ${ }^{[3]}$ is adopted, mainly including: physical function, physiological function, mental health, social function and emotional function, with 20 points for each item, and the score is positively correlated with the quality of life.

\subsection{Statistical treatments}

Software SPSS23.0 was selected as the tool. Measurement data is processed by test and expressed by $(\mathrm{x} \pm \mathrm{s})$, and counting data is processed by $\mathrm{X}^{2}$ test and expressed by $\%$. $\mathrm{P}<0.05$, which was statistically significant.

\section{Results}

\subsection{Comparison of the incidence of adverse reactions to radiotherapy}

After nursing, the incidence of adverse radiation reactions in group B was lower than that in group A, with statistical significance $(\mathrm{P}<0.05)$.

Table 1. Incidence of complications (n, \%)

\begin{tabular}{ccccc}
\hline Group & Pulmonary infection & Electrolyte imbalance & Lose weight & Total incidence rate \\
\hline Group A $(\mathbf{n}=\mathbf{4 8})$ & $1(2.08)$ & $3(6.25)$ & $3(6.25)$ & $7(14.58)$ \\
Group B $(\mathbf{n}=\mathbf{4 8})$ & $0(0.00)$ & $0(0.00)$ & $1(2.08)$ & $1(2.08)$ \\
$\mathbf{X}^{\mathbf{2}}$ value & - & - & - & 5.000 \\
P value & - & - & - & 0.025 \\
\hline
\end{tabular}




\subsection{Comparison of clinical index}

Before nursing, there was no significant difference in serum albumin, hemoglobin and lymphocyte coefficient between the two groups, without statistical significance $(P>0.05)$. After nursing, the serum albumin, hemoglobin and lymphocyte coefficients in group B were significantly higher than those in group A, with statistical significance $(\mathrm{P}<0.05)$.

Table 2. Clinical indexes $(\mathrm{x} \pm \mathrm{s})$

\begin{tabular}{ccccccc}
\hline & \multicolumn{3}{c}{ Before nursing } & \multicolumn{3}{c}{ After nursing } \\
\cline { 2 - 6 } Group & $\begin{array}{c}\text { Serum } \\
\text { albumin }(\mathbf{g} / \\
\mathbf{L})\end{array}$ & $\begin{array}{c}\text { Hemoglobin } \\
(\mathbf{g} / \mathbf{L})\end{array}$ & $\begin{array}{c}\text { Lymphocyte } \\
\text { coefficient }\left(\mathbf{1 0}^{\mathbf{9}} / \mathbf{L}\right)\end{array}$ & $\begin{array}{c}\text { Serum } \\
\text { albumin }(\mathbf{g} / \\
\mathbf{L})\end{array}$ & $\begin{array}{c}\text { Hemoglobin } \\
(\mathbf{g} / \mathbf{L})\end{array}$ & $\begin{array}{c}\text { Lymphocyte } \\
\text { coefficient } \\
(\mathbf{1 0} / \mathbf{L})\end{array}$ \\
\hline $\begin{array}{c}\text { Group A } \\
(\mathbf{n = 4 8 )}\end{array}$ & $98.57 \pm 0.17$ & $21.75 \pm 0.56$ & $1.08 \pm 0.45$ & $109.36 \pm 0.62$ & $28.36 \pm 0.67$ & $1.32 \pm 0.65$ \\
Group B & $98.54 \pm 0.18$ & $21.69 \pm 0.54$ & $1.07 \pm 0.51$ & $121.52 \pm 0.74$ & $37.28 \pm 0.84$ & $1.72 \pm 0.45$ \\
$(\mathbf{n = 4 8})$ & & & & & & \\
$\mathbf{t}$ value & 0.839 & 0.534 & 0.102 & 87.266 & 57.516 & 3.505 \\
P value & 0.403 & 0.594 & 0.919 & 0.000 & 0.000 & 0.001 \\
\hline
\end{tabular}

\subsection{Comparison of quality of life}

The quality of life of physical function, physiological function, mental health, social function and emotional function in group B was significantly higher than that in group A, with statistical significance $(\mathrm{P}<0.05)$.

Table 3. Quality of life ( $x \pm s$, points)

\begin{tabular}{cccccc}
\hline Group & $\begin{array}{c}\text { Physical } \\
\text { function }\end{array}$ & $\begin{array}{c}\text { Physiological } \\
\text { function }\end{array}$ & $\begin{array}{c}\text { Mental } \\
\text { health }\end{array}$ & $\begin{array}{c}\text { Social } \\
\text { function }\end{array}$ & $\begin{array}{c}\text { Emotional } \\
\text { function }\end{array}$ \\
\hline Group A $(\mathbf{n}=\mathbf{4 8})$ & $16.48 \pm 1.42$ & $16.57 \pm 1.56$ & $16.87 \pm 1.68$ & $16.54 \pm 1.59$ & $16.59 \pm 1.52$ \\
Group B $(\mathbf{n}=\mathbf{4 8})$ & $18.25 \pm 0.47$ & $18.45 \pm 0.49$ & $18.64 \pm 0.12$ & $18.54 \pm 0.36$ & $18.57 \pm 0.17$ \\
t value & 8.198 & 7.966 & 7.281 & 8.500 & 8.969 \\
P value & 0.000 & 0.000 & 0.000 & 0.000 & 0.000 \\
\hline
\end{tabular}

\section{Discussion}

Esophageal cancer is a disease with high morbidity and mortality. According to the relevant investigation ${ }^{[4]}$, the incidence rate of esophageal cancer ranks sixth in all malignant tumors, and the mortality rate ranks fourth. After comprehensive treatment, the 5-year survival rate is only $20 \%$. Therefore, radiotherapy can effectively control the patient's condition, but because of the poor nutritional state of the patients, the body's tolerance to chemotherapy is low. Thus, it can increase the risk of clinical treatment. Hence, how to effectively improve the nutritional status of patients and improve the quality of life of patients is one of the primary clinical problems. With the continuous development of medical research, some scholars pointed out that ${ }^{[5]}$ predictive dietary guidance can effectively improve the diet of patients with esophageal cancer and further improve their nutritional indicators, with ideal results. Therefore, in order to further explore its clinical application value, our hospital carried out this study.

The results of this study reveal that when predictive diet guidance is applied to esophageal cancer patients undergoing radiation, the incidence of radiotherapy adverse responses is lower in group B, clinical indicators are greater in group B, and quality of life is higher in group B. The impact of the application is 
significant. Predictive dietary guidance may increase patients' tolerance to radiation and successfully limit the harm caused by radiotherapy to the patient's body through dietary guidance before to treatment, which is conducive to radiotherapy. Nutritional support, nasal feeding, and other interventions are offered as the patient's condition progresses during radiation. It is capable of efficiently maintaining a patient's regular nutritional supply. Following radiation, dietary advice can help patients' esophageal mucosa repair, reducing the chance of additional esophageal injury and raising the risk of radiotherapy-related side effects. As a result, predictive dietary guidance can help patients improve their physical fitness, improve their quality of life, and have a favorable influence on their clinical therapy ${ }^{[6-7]}$.

In summary, predictive dietary guidance can significantly improve the nutritional status of patients and reduce adverse reactions of radiotherapy. It has significant effect and high clinical application value, and can be popularized.

\section{Disclosure statement}

The author declares no conflict of interest.

\section{References}

[1] Liu L, 2020, Application Effect of Whole-course High-quality Nursing in Patients with Esophageal Cancer. International Journal of Nursing, 39(10): 1853-1856.

[2] Qian J, Zhu Y, 2021, The Application Effect of Targeted Nursing in Reducing the Infection Rate of Long-term Nasogastric Feeding Patients with Esophageal Cancer. China Medical Herald, 18(25): 190192, 196.

[3] Chen J, 2017, Application and Clinical Nursing of PICC Catheterization in Patients with Esophageal Cancer after Operation. Guide of China Medicine, 30(12): 126-128.

[4] Huang W, Liu L, 2020, Clinical Application of Whole-process High-quality Nursing in the Nursing of Patients with Esophageal Cancer. Journal of Clinic Nursing's Practicality, 5(22): 40.

[5] Ni J, Zhang Q, 2016, Application of Rapid Rehabilitation Surgical Nursing in Perioperative Period of Minimally Invasive Esophageal Cancer Patients. Nursing Practice and Research, 13(24): 50-51.

[6] Liang W, Huang H, Chen X, et al., 2006, Application and Nursing of Total Parenteral Nutrition in Patients undergoing Esophageal Surgery. Journal of Qilu Nursing, 12(8): 763-764.

[7] Hao J, 2016, Clinical Effect of Comfort Nursing on Patients in Interventional Treatment of Esophageal Cancer Patients. Modern Digestion \& Intervention, 21(6): 924-926.

\section{Publisher's note}

Bio-Byword Scientific Publishing remains neutral with regard to jurisdictional claims in published maps and institutional affiliations. 